\title{
Fixed Point and Best Proximity Theorems under Two Classes of Integral-Type Contractive Conditions in Uniform Metric Spaces
}

\author{
M. De la Sen \\ Institute of Research and Development of Processes, University of the Basque Country, \\ Campus of Leioa (Bizkaia), P.O. Box. 644- Bilbao, 48080 Bilbao, Spain \\ Correspondence should be addressed to M. De la Sen, manuel.delasen@ehu.es \\ Received 5 July 2010; Accepted 14 October 2010 \\ Academic Editor: S. Reich \\ Copyright (C) 2010 M. De la Sen. This is an open access article distributed under the Creative \\ Commons Attribution License, which permits unrestricted use, distribution, and reproduction in \\ any medium, provided the original work is properly cited. \\ This paper investigates the existence of fixed points and best proximity points of $p$-cyclic self- \\ maps on a set of subsets of a certain uniform space under integral-type contractive conditions. The \\ parallel properties of the associated restricted composed maps from any of the subsets to itself are \\ also investigated. The subsets of the uniform space are not assumed to intersect.
}

\section{Introduction}

Fixed point theory is of an intrinsic theoretical interest but also a useful tool in a wide class of practical problems. There is an exhaustive variety of results concerning fixed point theory in Banach spaces and metric spaces involving different types of contractive conditions including those associated with the so-called Kannan maps and with Meir-Keeler contractions (see, e.g., [1-6]). There is also a rich background literature concerning the use of contractive conditions in integral form using altering distances, Lebesgue integrable test functions, and comparison functions, [7-9]. Also, the so-called reasonable expansive mappings have been investigated in [10], and conditions for the existence of fixed points have been given. It has been used, for instance, for the study of the Lyapunov stability of delay-free dynamic systems and also for that of dynamic systems subject to delays and then described by functional differential equations (see, for instance, $[11,12]$ ) concerning a related fixed point background for those systems and [12-15] concerning some related background for stability. On the other hand, it has also been useful for investigating the stability of hybrid systems consisting of coupled continuous-time and discrete-time or digital dynamic subsystems [16]. This paper considers $p$-cyclic self-maps in a uniform space $(X, \Phi)$, where $X$ is a nonempty set equipped with a nonempty family $\Phi$ of subsets of $X \times X$ satisfying certain uniformity properties. The family $\Phi$ 
is called the uniform structure of $X$, and its elements are called entourages, neighbourhoods, or surroundings. The uniform space $(X, \Phi)$ is assumed to be endowed with an $A$-distance or and $E$-distance. The existence of fixed points and best proximity points in restricted $p$-cyclic self-maps $F: X\left|\left(\bigcup_{i \in \bar{p}} A_{i}\right) \rightarrow X\right|\left(\bigcup_{i \in \bar{p}} A_{i}\right)$ of $X$, [8], subject to the constraint $F\left(A_{i}\right) \subseteq A_{i+1}$ for each pair of adjacent subsets $A_{i}$ of $X$, for all $i \in \bar{p}:=\{1,2, \ldots, p\}, p \geq 2$, under the cyclic condition $A_{p+i}=A_{i}$; for all $i \in \mathbf{Z}_{+}$, is investigated separately under two groups of integraltype contractive conditions. One of such groups involves a positive integrand test function while the other combines a positive integrand with a comparison function. Some properties of the composed restricted self-maps on each of the subsets are also investigated. The subsets of the uniform structure do not necessarily intersect. If the sets do not intersect, then it is proven that $g=g_{i} \equiv g_{i i+1}:=\operatorname{dist}\left(A_{i}, A_{i+1}\right)=d\left(z_{i}, F z_{i}\right)$ if $(X, d)$ is a metric space endowed with a distance map $d: X \times X \rightarrow \mathbf{R}_{0+}$, some $z_{i} \in A_{i}$, for all $i \in \bar{p}$, and $z_{i}$ is said to be a best proximity point. Also, it follows that $g=g_{i j}:=\operatorname{dist}\left(A_{i}, A_{j}\right)=d\left(z_{i}, F^{j-i} z_{i}\right)$, for all $i, j(>i) \in \bar{p}$ for some $z_{i} \in A_{i}$, for all $i \in \bar{p}$. If the self-map $T$ of $X$ is nonexpanding, then $g=g_{i i+1}:=$ $\operatorname{dist}\left(A_{i}, A_{i+1}\right)>0$, for all $i \in \bar{p}$, [8].

\section{Basic Results about $A$-Distances, $E$-Distances, and $V$-Closeness}

Define the nonempty family $\Phi$ of subsets of $X \times X$ of the form $\Phi:=\left(\bigcup_{i, j \in \bar{p}} \Phi_{i j}\right)$ with $\Phi_{i j}:=$ $A_{i} \times A_{j}$, for all $i, j \in \bar{p}:=\{1,2, \ldots, p\}$. Note by construction that

$$
\begin{aligned}
V \in \Phi \Longrightarrow & {\left[\left(V \in A_{i} \times A_{j} ; \forall i, j \in \bar{p}\right) \vee V \in\left(\bigcup_{i \in p_{x}} A_{i}\right) \times\left(\bigcup_{i \in p_{y}} A_{j}\right)\right.} \\
& \left.\quad \text { for some nonempty finite subsets of positive integers } p_{x}, p_{y} \subseteq \bar{p}\right] .
\end{aligned}
$$

The following definitions of $V$-closeness and an $A$ and $E$-distances are used throughout the paper.

Definition 2.1 (see [7,9]). If $V \in \Phi$ and $(x, y) \in V$ and $(y, x) \in V$, then $x$ and $y$ are said to be $V$-close. A sequence $\left\{x_{n}\right\}_{0}^{\infty} \subset X$ is a Cauchy sequence for $\Phi$ if for any $V \in \Phi$, there exists $N \geq 1$ such that $x_{n}$ and $x_{m}$ are $V$-close for $n, m \geq N$.

Definition 2.2. A function $d: X \times X \rightarrow \mathbf{R}_{0+}$ is said to be an $A$-distance if

(1) $d(x, y)=0 \Leftrightarrow x=y$ for all $x, y \in X$;

(2) for each $V \in \Phi$, there exist $\delta=\delta(V)>0$ such that

$$
\max (d(z, x), d(z, y)) \leq \delta \quad \text { for some } z \in X \Longrightarrow(x, y) \in V \text {. }
$$

Definition 2.2 generalizes slightly that of [7] by admitting $\delta$ to depend on $V$ since it is being used on distinct sets $A_{i}, i \in \bar{p}$. Note that $V \in \Phi$ is symmetrical, that is, $V=$ $V^{-1}=\{(y, x):(x, y) \in V\}$ then $(x, y) \in V \Leftrightarrow(x, y) \in V$ so that $x$ and $y$ are $V$-close under Definition 2.2. 
Definition 2.3 (see [7]). A function $d: X \times X \rightarrow \mathbf{R}_{0_{+}}$is said to be an $E$-distance if

(1) it is an $A$-distance;

(2) $d(x, y) \leq d(x, z)+d(z, y)$; for all $x, y, z$.

Assertion 1. Assume that any $Z \in \Psi \subset X \times X$ is symmetrical, that is, $Z=Z^{-1}=\{(y, x) \mid$ $(x, y) \in Z$. Then, $d: X \times X \rightarrow \mathbf{R}_{0+}$ is an $A$-distance if and only if $x$ and $y$ are $Z$-close for all $Z \in \Psi$ provided that $\max (d(z, x), d(z, y))<\delta$ for some $z \in X$ and some $\delta>0$.

Proof. It follows from the symmetry of all $Z \in \Psi$ and Definition 2.3 by a simple contradiction argument. Take a pair $(x, y) \in Z$ from Definition 2.3 since $d: X \times X \rightarrow \mathbf{R}_{0+}$ is an $A$-distance fulfilling $\max (d(z, x), d(z, y))<\delta$ for some $z \in X$ and some $\delta>0$. Such a pair always exists for any $Z \in \Psi$. Since $V$ is symmetrical, then $(y, x) \in Z$. Since $(x, y) \in Z$ if and only if $(y, x) \in Z$ then $x$ and $y$ are $V$-close.

Assertion 2. (1) $\Phi_{i j}$ and $\Phi:=\left(\bigcup_{i, j \in \bar{p}} \Phi_{i j}\right)$ are symmetrical.

(2) If $V \in \Phi$ is of the form $V_{X} \times V_{X}$ then $V$ is symmetrical.

(3) If $V \subseteq \bigcup_{i \in p_{x}, j \in p_{y}} \Phi_{i j}$ is nonempty and $p_{x}, p_{y} \subset \bar{p}$ with $p_{x} \cap p_{y}=\emptyset$, then $V$ is not symmetrical.

If, in-addition, $A_{i} \cap A_{j}=\emptyset$, for all $i, j \in \bar{p}$ then there are no $x, y$ in $\Phi$ being $V$-close.

Proof. (1) $\left[(x, y) \in \Phi_{i j}=A_{i} \times A_{j} \Leftrightarrow\left(x \in A_{i}, y \in A_{j}\right) \Leftrightarrow(y, x) \in \Phi_{j i}=A_{j} \times A_{i}\right] \Leftrightarrow \Phi_{i j}$ is symmetrical. $(x, y) \in \Phi \Leftrightarrow x, y \in \bigcup_{i \in \bar{p}} A_{i} \Leftrightarrow(y, x) \in \Phi \Leftrightarrow \Phi$ is symmetrical. Assertion 2(1) has been proven.

(2) $(x, y) \in V \in \Phi \Rightarrow\left((x, y) \in\left(\bigcup_{i \in p_{x}} A_{i}\right) \times\left(\bigcup_{i \in p_{x}} A_{i}\right) \cap V\right) \Leftrightarrow(y, x) \in\left(\bigcup_{i \in p_{x}} A_{i}\right) \times$ $\left(\bigcup_{i \in p_{x}} A_{i}\right) \cap V$ for some $p_{x} \subseteq \bar{p} \Longrightarrow V$ is symmetrical. Assertion 2 (2) is proven .

(3) Proceed by contradiction: $V$ symmetrical $\Leftrightarrow\left[(x, y) \in V \Rightarrow\left((x, y) \in \bigcup_{i \in p_{x}, j \in p_{y}} \Phi_{i j}=\right.\right.$ $\left.\left.\bigcup_{i \in p_{x}} A_{i} \times \bigcup_{j \in p_{y}} A_{j} \equiv \bigcup_{i \in p_{y}} A_{i} \times \bigcup_{j \in p_{x}} A_{j} \ni(y, x) \Leftarrow(y, x) \in V\right)\right] \Rightarrow p_{x} \cap p_{y} \neq \emptyset$ what contradicts $p_{x} \cap p_{y}=\emptyset$.

Assertion 2 states that some, but not all, nonempty subsets $V$ of $\emptyset$ are symmetrical. For instance, if $V=\left(A_{1} \cup A_{2}\right) \times\left(A_{3} \cup A_{4}\right)$, then $V$ is not symmetrical since there are $(x, y) \in V$ such that $(y, x)$ are not in $V$; that is, there are pairs $x, y$ which are not $V$-close. If furthermore the sets $A_{(\cdot)}$ are disjoint, then there is no pair in $\Phi$ being $V$-close (Assertion 2(3)). Note that under symmetry of $V$, the second property of an $A$-distance can be rewritten in an equivalent form by replacing $(x, y) \in V$ with $(x, y)$ being $V$-close. The subsequent result states that, contrarily to results in former studies related to $A$ and $E$-distances $[7,9]$, the second property guaranteeing an $A$-distance necessarily involves $\delta$-values exceeding distances between the various subsets $A_{i}, i \in \bar{p}$.

Lemma 2.4. Assume that $d: X \times X \rightarrow \mathbf{R}_{0+}$ is an A-distance and $V \subseteq \Phi_{i j} \in \Phi$ for some $i, j \in \bar{p}$. If $(x, y) \in V$, then $\max (d(z, x), d(z, y))<\delta_{i j}$ for some $z \in X$ and some $\delta_{i j}>\operatorname{dist}\left(A_{i}, A_{j}\right)$.

Proof. Assume that $(x, y) \in V$, so that $x \in A_{i}$ and $y \in A_{j}$, and $\max (d(z, x), d(z, y))<$ $\operatorname{dist}\left(A_{i}, A_{j}\right)$, for some $z \in X$. The following cases can occur.

(1) If $z=x \in A_{i}$, and since $y \in A_{j}$, then

$$
0 \leq \operatorname{dist}\left(A_{i}, A_{j}\right) \leq d(z, y)=\max (d(x, x), d(z, y))<\operatorname{dist}\left(A_{i}, A_{j}\right),
$$

which leads to the contradiction $\operatorname{dist}\left(A_{i}, A_{j}\right)<\operatorname{dist}\left(A_{i}, A_{j}\right)$. 
(2) If $z(\neq x) \in A_{i}$, and since $y \in A_{j}$, then

$0 \leq \operatorname{dist}\left(A_{i}, A_{j}\right) \leq \max \left(d(z, x), \operatorname{dist}\left(A_{i}, A_{j}\right)\right) \leq \max (d(z, x), d(z, y))<\operatorname{dist}\left(A_{i}, A_{j}\right)$,

which leads to the same contradiction as in Case (1).

(3) If $z(=y) \in A_{j}$ and if $z(\neq y) \in A_{j}$, the above contradiction of cases (1) and (2), is also obtained by replacing $A_{i} \leftrightarrow A_{j}$.

(4) If $z \in \overline{A_{i} \cup A_{j}} \cap X$, then

$0 \leq \operatorname{dist}\left(A_{i}, A_{j}\right) \leq \max \left(d(z, x), \operatorname{dist}\left(A_{i}, A_{j}\right)\right) \leq \max (d(z, x), d(z, y))<\operatorname{dist}\left(A_{i}, A_{j}\right)$

which leads to the same contradiction as that of case (1).

The following lemma is a direct consequence of Lemma 2.4:

Lemma 2.5. Assume that $d: X \times X \rightarrow \mathbf{R}_{0+}$ is an A-distance and $V \subseteq \bigcup_{i \in p_{x}, j \in p_{y}} \Phi_{i j}$ for $p_{x}, p_{y} \subset \bar{p}$. If $(x, y) \in V$ then $\max (d(z, x), d(z, y))<\delta$ for some $z \in X$ and some $\delta>\max _{i \in p_{x}, j \in p_{y}} \operatorname{dist}\left(A_{i}, A_{j}\right)$.

\section{Main Results about Fixed Points and Best Proximity Points}

Consider $p$-cyclic self-maps $F: X\left|\left(\bigcup_{i \in \bar{p}} A_{i}\right) \rightarrow X\right|\left(\bigcup_{i \in \bar{p}} A_{i}\right)$ subject to $F\left(A_{i}\right) \subseteq$ $A_{i+1}$; for all $i \in \bar{p}=\{1,2, \ldots, p\}$. The objective is to first investigate if each of them has a fixed point and then if they have a common fixed point through contraction conditions on Lebesgue integrals and use of comparison functions. Without loss of generality, we discuss the fixed points of self-maps $F$ of $X$. Consider a Lebesgue-integrable map $\varphi: \mathbf{R}_{0+} \rightarrow \mathbf{R}_{0+}$ which satisfies $\int_{0}^{\varepsilon} \varphi(t) d t>0$, for all $\varepsilon \in \mathbf{R}_{+}$such that for all $x \in A_{i}$, for all $y \in A_{i+1}$.

Define also the composed self-map $F^{p}: X\left|\bigcup_{i=1}^{p} A_{i} \rightarrow X\right| \bigcup_{i=1}^{p} A_{i}$ as $\bigcup_{i=1}^{p} A_{i} \ni y=$ $F^{p} x \in \bigcup_{i=1}^{p} A_{i}$ from the self-map $F: X\left|\bigcup_{i=1}^{p} A_{i} \rightarrow X\right| \bigcup_{i=1}^{p} A_{i}$ whose restrictions to $A_{i}$, $F^{p}: X\left|A_{i} \rightarrow X\right| A_{i}$; for all $i \in \bar{p}$, are defined via the restriction $F^{p}\left|A_{i}:=F_{p-i}\right| A_{p-i} \circ \cdots \circ$ $F_{i+1}\left|A_{i+1} \circ F_{i}\right| A_{i}$; for all $i \in \bar{p}$ by $y=F^{p} x \in A_{i}$ for each $x \in A_{i}$; for all $i \in \bar{p}$. Note that the domain of the self-map $F^{p}$ of $X$ is $\bigcup_{i=1}^{p} A_{i}$ while that of $F^{p} \mid A_{i}$ is $A_{i}$. The paper investigates, under two types of integral-type contractive conditions of self-maps $F$ of $X$, the existence of fixed points of such a self-map in $\bigcap_{i=1}^{p} A_{i}$, provided that the intersection is nonempty. In that case, the fixed points coincide with those of the self-map $F^{p}: X\left|\bigcup_{i=1}^{p} A_{i} \rightarrow X\right| \bigcup_{i=1}^{p} A_{i}$. It also investigated the existence of best proximity points between adjacent and nonadjacent subsets $A_{i}$; for all $i \in \bar{p}$ for the case that $\bigcap_{i=1}^{p} A_{i}=\emptyset$. In such a case, the best proximity points at each pair of adjacent subsets $A_{i}, A_{i+1}$; for all $i \in \bar{p}$ are also fixed points of the composed self-maps $F^{p}: X\left|A_{i} \rightarrow X\right| A_{i}$ from each subset $A_{i}$ to itself; for all $i \in \bar{p}$ even under weaker contractive integral-type conditions. A key basic result used in the mathematical proofs is that the distance between any pair of (adjacent or nonadjacent) subsets is identical for nonexpansive contractions.

It is first assumed that the integral-type contractive Condition 1 below holds. 
Condition 1. One has

$$
\int_{0}^{d\left(F^{n p+j+1} x, F^{n p+j+1} y\right)} \varphi(t) d t \leq\left(1-\alpha_{j n}\right) \int_{0}^{d\left(F^{n p+j} x, F^{n p+j} y\right)} \varphi(t) d t+\alpha_{j n} \int_{0}^{\operatorname{dist}\left(A_{j}, A_{j+1}\right)} \varphi(t) d t ; \quad \forall j \in \bar{p},
$$

where $\left\{\alpha_{j n}\right\}_{n=1}^{\infty}$ are sequences of nonnegative real numbers subject to $\sum_{n=1}^{\infty} \alpha_{j n}=\infty$; for all $j \in \bar{p}$, for all $n \in \mathbf{Z}_{+}$. The self-map $F$ of $X$ is said to be reasonably nonexpansive through this paper if

$$
\begin{gathered}
\int_{0}^{d\left(F^{n p} x, F^{n p} y\right)} \varphi(t) d t \leq K_{M} \int_{0}^{d(x, y)} \varphi(t) d t+K_{M}^{\prime} \\
d\left(F^{i p} x, F^{i p} y\right) \leq K_{M} d(x, y)+K_{M}^{\prime} \quad \forall x, y \in X ; \forall n \in \mathbf{Z}_{+}
\end{gathered}
$$

for some nonnegative real constants $K_{M}$ and $K_{M}^{\prime}$. In particular, $F$ is reasonably nonexpansive if $F^{p}$ is nonexpansive. The following result follows from Condition 1.

Theorem 3.1. The following properties hold under Condition 1 for any A-distance $d: X \times X \rightarrow \mathbf{R}_{0+}$ :

(i) The restricted self-maps $F^{p} \mid A_{i}$; for all $i \in \bar{p}$ satisfying (3.1) are all nonexpansive, and so it is the self-map $F: X\left|\bigcup_{i=1}^{p} A_{i} \rightarrow X\right| \bigcup_{i=1}^{p} A_{i}$;

(ii) $\infty>\operatorname{dist}\left(A_{i}, A_{i+1}\right)=g \geq 0$; for all $i \in \bar{p}$;

(iii) $\limsup _{n \rightarrow \infty} \int_{0}^{d\left(F^{(n+1) p} x, F^{(n+1) p} y\right)} \varphi(t) d t \leq M<\infty$, for all $(x, y) \in A_{i} \times A_{i+1}$; for all $i \in \bar{p}$, with $M=M(g)$ and $M: \mathbf{R}_{0+} \rightarrow \mathbf{R}_{0+}$ being monotone increasing with $M(0)=0$;

(iv) If $\operatorname{dist}\left(A_{i}, A_{i+1}\right)=0$; for all $i \in \bar{p}$, that is, $\bigcap_{i \in \bar{p}} A_{i} \neq \emptyset$, then there is a fixed point $\bar{x} \in$ $\left(\bigcap_{i \in \bar{p}} A_{i}\right) \cap \operatorname{Fix}\left(F^{p}\right)$ of the self-map $F^{p}$ of $X$ and of its restrictions to $\bigcup_{i \in \bar{p}} A_{i}$ and $\bigcap_{i \in \bar{p}} A_{i}$ defined through the natural set inclusions $\bigcap_{i \in \bar{p}} A_{i} \subseteq \bigcup_{i \in \bar{p}} A_{i} \subset X$. Also, $\bar{x} \in \operatorname{Fix}(F)$ for the self-map $F: X\left|\bigcup_{i=1}^{p} A_{i} \rightarrow X\right| \bigcup_{i=1}^{p} A_{i}$.

Proof. Consider some $A$-distance $d: X \times X \rightarrow \mathbf{R}_{0+}$. Note that for each

$$
V \subseteq\left(\bigcup_{i \in \bar{p}} A_{i}\right) \times\left(\bigcup_{i \in \bar{p}} A_{i}\right) \in \Phi, \quad \exists \delta=\delta(V)>\max _{i \in \bar{p}} \operatorname{dist}\left(A_{i}, A_{i+1}\right) \geq 0 ; \quad \forall i \in \bar{p}
$$

such that

$$
\begin{aligned}
\max & (d(z, x), d(z, y)) \leq \delta \quad \text { for some } z \in \bigcup_{i \in \bar{p}} A_{i} \subset X \\
& \Longrightarrow[(x, y) \in V \wedge(y, x) \in V] \\
& \Longleftrightarrow x, y \text { are } V \text {-close }
\end{aligned}
$$

If, in particular, $V \subseteq A_{i} \times A_{i+1}$; for all $i \in \bar{p}$, then $(x, y) \in V$ if $\max (d(z, x), d(z, y)) \leq \delta$ with any $\delta>\operatorname{dist}\left(A_{i}, A_{i+1}\right) \geq 0$ and $z \in A_{i} \cup A_{i+1}$; for all $i \in \bar{p}$, and if $V \subseteq\left(A_{i} \cup A_{i+1}\right) \times\left(A_{i} \cup A_{i+1}\right)$ 
then $[(x, y) \in V \wedge(y, x) \in V] \Leftrightarrow x, y$ are $V$-close. It is first proven that the self-map $F^{p}$ of $X$ satisfying (3.1) is nonexpansive. Proceed by contradiction by assuming that it is expansive. Then, one gets the following by defining a real sequence $\left\{\alpha_{n}\right\}_{n=1}^{\infty}$ with general term $\alpha_{n}:=$ $1-\prod_{j=1}^{n}\left[1-\alpha_{j n}\right] \in[0,1]$ :

$$
\begin{aligned}
& \left(\prod_{j=1}^{p}\left[1-\alpha_{j n}\right]\right) \int_{0}^{d\left(F^{n p} x, F^{n p} y\right)} \varphi(t) d t+\sum_{\ell=1}^{p} \prod_{j=\ell+1}^{p}\left(\left[1-\alpha_{j n}\right]\right) \alpha_{\ell n} \int_{0}^{\operatorname{dist}\left(A_{\ell}, A_{\ell+1}\right)} \varphi(t) d t \\
& \quad \geq \alpha_{n} \int_{0}^{d\left(F^{n p} x, F^{n p} y\right)} \varphi(t) d t+\min _{j \in \bar{p}}\left(1-\alpha_{n}\right) \int_{0}^{\operatorname{dist}\left(A_{j}, A_{j+1}\right)} \varphi(t) d t \\
& \quad \geq \int_{0}^{d\left(F^{(n+1) p} x, F^{(n+1) p} y\right)} \varphi(t) d t>\int_{0}^{d\left(F^{n p} x, F^{n p} y\right)} \varphi(t) d t \\
& \Longrightarrow \min _{j \in \bar{p}} \int_{0}^{\operatorname{dist}\left(A_{j}, A_{j+1}\right)} \varphi(t) d t>\int_{0}^{d\left(F^{n p} x, F^{n p} y\right)} \varphi(t) d t \\
& \Longrightarrow \min _{j \in \bar{p}} \operatorname{dist}\left(A_{j}, A_{j+1}\right)>d\left(F^{n p} x, F^{n p} y\right) \equiv d\left(x_{1}, y_{1}\right)
\end{aligned}
$$

for some $x_{1}, y_{1} \in \bigcup_{i=1}^{p} A_{i}$ which is a contradiction, and the self-map $F^{p}$ (and then the selfmap $F$ ) of $X$ is nonexpansive and property (i) holds. Now, its is proven by contradiction that $\operatorname{dist}\left(A_{i}, A_{i+1}\right)=g \geq 0$, for all $i \in \bar{p}$. Assume that there exist $i, j \in \bar{p}$ satisfying $1 \leq j \leq p-i$ such that $\operatorname{dist}\left(A_{i}, A_{i+1}\right)>\operatorname{dist}\left(A_{i+j}, A_{i+j+1}\right)$. Then there are best proximity points $z_{i} \in A_{i}, \xi_{i+j} \in A_{i+j}$ and some $\bar{z}_{i} \in A_{i}$ such that, since $p>j$ and the self-map $F$ of $X$ is nonexpanding, one gets

$$
\begin{aligned}
d\left(z_{i}, F z_{i}\right) & =\operatorname{dist}\left(A_{i}, A_{i+1}\right)>\operatorname{dist}\left(A_{i+j}, A_{i+j+1}\right)=d\left(\xi_{i+j}, F \xi_{i+j}\right) \\
& =d\left(F^{j} \bar{z}_{i}, F^{j+1} \bar{z}_{i}\right) \geq d\left(F^{p} \bar{z}_{i}, F^{p+1} \bar{z}_{i}\right)=d\left(\widehat{z}_{i}, F \widehat{z}_{i}\right) \leq \operatorname{dist}\left(A_{i}, A_{i+1}\right) .
\end{aligned}
$$

for some $\widehat{z}_{i} \in A_{i}$ with the last inequality being strict unless $\widehat{z}_{i}=z_{i}$, what is a contradiction if $\widehat{z}_{i} \neq z_{i}$. Now, assume that $\widehat{z}_{i}=F^{p} \bar{z}_{i}=z_{i}$, then the best proximity point $z_{i}=\widehat{z}_{i} \in \operatorname{Fix}\left(F^{p}\right)$ since $F^{p} \widehat{z}_{i}=F^{2 p} \bar{z}_{i}=F^{p} z_{i}=\widehat{z}_{i}=z_{i}$ and $\operatorname{dist}\left(A_{i}, A_{i+1}\right)=0$, that is, $A_{i} \cap A_{i+1} \neq \emptyset$. This is a contradiction to the assumption $\operatorname{dist}\left(A_{i}, A_{i+1}\right)>\operatorname{dist}\left(A_{i+j}, A_{i+j+1}\right)$. Then, $\operatorname{dist}\left(A_{i}, A_{i+1}\right)=g \geq 0$, for all $i \in \bar{p}$ and $\bigcap_{i=1}^{p} A_{i} \neq \emptyset$ if and only if $g=0$. Since the self-map $F^{p}$ of $X$ restricted to $\bigcup_{i=1}^{p} A_{i}$ is nonexpansive, then the self-map $F$ of $X$ restricted to $\bigcup_{i=1}^{p} A_{i}$ is reasonably nonexpansive. It also follows by contradiction that $g<\infty$. Assume that $g=\infty$. Then, the following contradiction follows from (3.1):

$$
\begin{aligned}
0 \leq & \int_{0}^{d\left(F^{(n+1) p} x, F^{(n+1) p} y\right)} \varphi(t) d t \leq\left(\prod_{j=1}^{p}\left[1-\alpha_{j n}\right]\right) \int_{0}^{d\left(F^{n p} x, F^{n p} y\right)} \varphi(t) d t \\
& +\sum_{\ell=1}^{p} \prod_{j=\ell+1}^{p}\left(\left[1-\alpha_{j n}\right]\right) \alpha_{\ell n} \int_{0}^{\operatorname{dist}\left(A_{\ell}, A_{\ell+1}\right)} \varphi(t) d t<0
\end{aligned}
$$


for some $n \in \mathbf{Z}_{+}, j \in \bar{p}$, unless $\int_{0}^{d\left(F^{n p} x, F^{n p} y\right)} \varphi(t) d t=+\infty$ (and then $d\left(F^{n p} x, F^{n p} y\right)=$ $\infty$; for all $\left.x, y \in \bigcup_{i=1}^{p} A_{i}\right)$ provided that $\alpha_{j n}>0$. Such a $\alpha_{j n}$ always exists since $\sum_{n=1}^{\infty} \alpha_{j n}=\infty$; for all $j \in \bar{p}$. Then, $\infty>\operatorname{dist}\left(A_{i}, A_{i+1}\right)=g \geq 0$; for all $i \in \bar{p}$, and Property (ii) follows.

Note that (3.1) yields directly via recursion

$$
\begin{aligned}
& \int_{0}^{d\left(F^{(n+1) p} x, F^{(n+1) p} y\right)} \varphi(t) d t \\
& \leq\left(\prod_{j=1}^{p}\left[1-\alpha_{j n}\right]\right) \int_{0}^{d\left(F^{n p} x, F^{n p} y\right)} \varphi(t) d t+\sum_{\ell=1}^{p} \prod_{j=\ell+1}^{p}\left(\left[1-\alpha_{j n}\right]\right) \alpha_{\ell n} \int_{0}^{\operatorname{dist}\left(A_{\ell}, A_{\ell+1}\right)} \varphi(t) d t \\
& \leq \prod_{\ell=1}^{n-1}\left(\prod_{j=1}^{p}\left[1-\alpha_{j \ell}\right]\right) \int_{0}^{d\left(F^{p} x, F^{p} y\right)} \varphi(t) d t+\sum_{i=1}^{n-1}\left(\prod_{m=i+1}^{n-1}\left(\prod_{j=1}^{p}\left[1-\alpha_{j m}\right]\right)\right) \\
& \times\left(\sum_{\ell=1}^{p} \prod_{j=\ell+1}^{p}\left(\left[1-\alpha_{j i}\right]\right) \alpha_{\ell i} \int_{0}^{\operatorname{dist}\left(A_{\ell}, A_{\ell+1}\right)} \varphi(t) d t\right) ; \quad \forall n \in \mathbf{Z}_{+}, \forall x \in A_{i}, \forall y \in A_{i+1} .
\end{aligned}
$$

Note that $\rho_{n}:=\prod_{j=1}^{p}\left[1-\alpha_{j n}\right] \in[0,1]$. Define $\mathbf{Z}_{+}:=Z_{s} \cup Z_{c}$ with $Z_{s}:=\left\{n \in \mathbf{Z}_{+}: \rho_{n}<1\right\}, Z_{c}:=$ $\left\{n \in \mathbf{Z}_{+}: \rho_{n}=1\right\}$ and

$$
\begin{gathered}
\bar{\rho}:=\limsup _{m \rightarrow \infty}\left(\prod_{n=1}^{\mu\left(Z_{s m}\right)} \rho_{n}\right)^{1 / m} \\
Z_{s m}:=\left\{n \in \mathbf{Z}_{+}:\left[\rho_{n}<1 \wedge n \leq m\right]\right\} \subset Z_{s} \subseteq \mathbf{Z}_{+} .
\end{gathered}
$$

Note also that the cardinal (or discrete measure) of $Z_{s}$ is $\mu\left(Z_{s}\right)=\chi_{0}$ (i.e., infinity numerable), since otherwise, $\sum_{n=1}^{\infty} \alpha_{j n}<\infty$ for some $j \in \bar{p}$ (contrarily to one of the given hypothesis) and $\mu\left(Z_{c}\right) \leq \chi_{0}$. Since $\sum_{n=1}^{m} \alpha_{j n}=\infty$ and $\mu\left(Z_{s}\right)=\chi_{0}$, so that $\bar{\rho} \in(0,1)$, it follows that

$$
\lim _{m \rightarrow \infty} \prod_{n=1}^{m} \rho_{n}=\left(\prod_{n \in Z_{s}} \rho_{n}\right)\left(\prod_{n \in Z_{c}} \rho_{n}\right)=\left(\prod_{n \in Z_{s}} \rho_{n}\right)=\lim _{m \rightarrow \infty} \bar{\rho}^{m}=0 .
$$

Then, since the distance between any two adjacent sets $A_{i}, A_{i+1}$ is a real constant $g$, one gets the following from (3.8), and (3.10):

$$
\begin{aligned}
& \underset{n \rightarrow \infty}{\limsup } \int_{0}^{d\left(F^{(n+1) p} x, F^{(n+1) p} y\right)} \varphi(t) d t \\
& \quad=\limsup _{n \rightarrow \infty} \sum_{i=1}^{n-1}\left(\prod_{m=i+1}^{n-1}\left(\prod_{j=1}^{p}\left[1-\alpha_{j m}\right]\right)\right)\left(\sum_{\ell=1}^{p} \prod_{j=\ell+1}^{p}\left(\left[1-\alpha_{j i}\right]\right) \alpha_{\ell i} \int_{0}^{g} \varphi(t) d t\right) ; \\
& \leq M:=\frac{\bar{\alpha}}{1-\bar{\rho}}\left(\int_{0}^{g} \varphi(t) d t\right)<\infty ; \quad \forall x \in A_{i}, \forall y \in A_{i+1}, \forall i \in \bar{p},
\end{aligned}
$$


where $\bar{\alpha}:=\sup _{j \in \bar{p}, n \in \mathbf{Z}_{+}} \alpha_{j n} \leq 1$ since $\lim _{n \rightarrow \infty} \prod_{\ell=1}^{n-1}\left(\prod_{j=1}^{p}\left[1-\alpha_{j \ell}\right]\right)=\lim _{n \rightarrow \infty} \bar{\rho}^{n}=0$,

$$
\begin{aligned}
0 & \leq \sum_{i=1}^{n-1}\left(\prod_{m=i+1}^{n-1}\left(\prod_{j=1}^{p}\left[1-\alpha_{j m}\right]\right)\right)\left(\sum_{\ell=1}^{p} \prod_{j=\ell+1}^{p}\left(\left[1-\alpha_{j i}\right]\right) \alpha_{\ell i} \int_{0}^{g} \varphi(t) d t\right) \\
& <\infty ; \quad \forall x \in A_{i}, \forall y \in A_{i+1} .
\end{aligned}
$$

Note that $M=M(g)$ is monotone increasing with it argument $g$ and that $M(0)=0$. Property (iii) has been proven. If $g=0$, then $\bigcap_{i \in \bar{p}} A_{i} \neq \emptyset$ so that there is a fixed point $\bar{x}$ of $\widehat{F}_{1}^{p}: X \mid$ $\bigcap_{i \in \bar{p}} A_{i} \rightarrow X$ which is also a fixed point of its extensions $\hat{F}_{2}^{p}: X \mid \bigcup_{i \in \bar{p}} A_{i} \rightarrow X$ and $F^{p}: X \rightarrow$ $X$ since $F^{p}\left|\bigcup_{i \in \bar{p}} A_{i}=\widehat{F}_{2}^{p}, F^{p}\right| \bigcap_{i \in \bar{p}} A_{i}=\widehat{F}_{1}^{p}$ and $\widehat{F}_{2}^{p} \mid \bigcap_{i \in \bar{p}} A_{i}=\widehat{F}_{1}^{p}$. It turns out that $\bar{x}$ is also a fixed point of $F: X \mid \bigcup_{i=1}^{p} A_{i} \rightarrow \bigcup_{i=1}^{p} A_{i}$, [17]. Property (iv) has been proven.

Note that the proved boundedness property of the $A$-distance $\infty>\operatorname{dist}\left(A_{i}, A_{i+1}\right)$ also relies on the fact that this is a distance between best proximity points in adjacent sets. It is well known that a distance map in a metric space has always a uniform equivalent distance which is finite. The following two concluding results from (3.11) are direct since $\int_{0}^{\varepsilon} \varphi(t) d t>0$; for all $\varepsilon \in \mathbf{R}_{+}$.

Corollary 3.2. Assume that $g:=\operatorname{dist}\left(A_{i}, A_{i+1}\right)>0$; for all $i \in \bar{p}$ and that

$$
\exists \lim _{n \rightarrow \infty} \sum_{i=1}^{n-1}\left(\prod_{m=i+1}^{n-1}\left(\prod_{j=1}^{p}\left[1-\alpha_{j m}\right]\right)\right)\left(\sum_{\ell=1}^{p} \prod_{j=\ell+1}^{p}\left(\left[1-\alpha_{j i}\right]\right) \alpha_{\ell i} \int_{0}^{g} \varphi(t) d t\right)<\infty .
$$

Then, there is a set $S:=\left\{x_{i} \in A_{i}: i \in \bar{p}\right\}$ of card $S=p$ such that

$$
d\left(x_{i}, x_{i+1}\right)=\lim _{n \rightarrow \infty} d\left(F^{n p} x, F^{n p} y\right)=\frac{g \bar{\alpha}}{1-\bar{\rho}} ; \quad \forall(x, y) \in\left(\bigcup_{i \in \bar{p}} A_{i}\right) \times\left(\bigcup_{i \in \bar{p}} A_{i}\right) ; \forall i \in \bar{p}
$$

If $\bar{\alpha}=1-\bar{\rho}$, then the points of the set $S$ satisfy $g=d\left(x_{i}, x_{i+1}\right)$, for all $i \in \bar{p}$ so that $S$ is a set of best proximity points in $\bigcup_{i \in \bar{p}} A_{i}$ of the self-maps $F$ and $F^{p}$ of $X$. Each $x_{i} \in S$ is a fixed point of $F \mid A_{i}, a$ best proximity point of $F^{p} \mid A_{i}$; for all $i \in \bar{p}$ and satisfies $F x_{i}=x_{i+1}$ and $x_{i} \in \operatorname{Fix}\left(F^{p} \mid A_{i}\right)$ so that $F^{p} x_{i}=x_{i} ;$ for all $i \in \bar{p}$.

Corollary 3.3. If $g=0$ so that $\bigcap_{i \in \bar{p}} A_{i} \neq \emptyset$; for all $i \in \bar{p}$, then Corollary 3.2 still holds with the set $S$ consisting only of a set of identical points $\bar{x}$ in $\bigcap_{i \in \bar{p}} A_{i}$ such that $\bar{x} \in \operatorname{Fix}(F), \bar{x} \in \operatorname{Fix}\left(F^{p}\right)$, and $\bar{x} \in F^{p} \mid A_{i} ;$ for all $i \in \bar{p}$.

Since an $E$-distance is also an $A$-distance, the following conclusion is direct from Theorem 3.1 and Corollary 3.2.

Corollary 3.4. Theorem 3.1 and Corollary 3.2 also hold if $d: X \times X \rightarrow \mathbf{R}_{0+}$ is an E-distance.

An important relaxation of Condition 1 allows the reformulation of Theorem 3.1 and Corollaries 3.2-3.4 except in the result that $\bar{x} \in \operatorname{Fix}(F)$ when $g=0$ as follows. 
Corollary 3.5. Assume that Condition 1 is reformulated as the p-cyclic contractive Condition 2 below.

Condition 2. One has

$$
\int_{0}^{d\left(F^{(n+1) p} x, F^{(n+1) p} y\right)} \varphi(t) d t \leq\left(1-\alpha_{n}\right) \int_{0}^{d\left(F^{n p} x, F^{n p} y\right)} \varphi(t) d t+\alpha_{n} \min _{j \in \bar{p}} \int_{0}^{\operatorname{dist}\left(A_{j}, A_{j+1}\right)} \varphi(t) d t
$$

for a new real sequence $\left\{\alpha_{n}\right\}_{n=1}^{\infty}$ under the weaker constraints

$$
\alpha_{n}:=1-\prod_{j=1}^{p}\left[1-\alpha_{j n}\right] \in[0,1] ; \quad \forall n \in \mathbf{Z}_{+}, \quad \sum_{n=1}^{\infty} \alpha_{n}=+\infty
$$

and that the finite limit of Corollary 3.2 exists. Then, the following properties hold.

(i) Theorem 3.1 and Corollaries 3.2-3.4 still hold, except that in the case that the distance between adjacent sets $g$ is zero (i.e., if all subsets $A_{i}, i \in \bar{p}$ have a nonempty intersection), the property $\bar{x} \in \operatorname{Fix}(F)$ is not guaranteed, since the restricted selfmaps $F: X\left|A_{i} \rightarrow X\right| A_{i+1}$ can be expansive for some $i \in \bar{p}$.

(ii) If $g>0$ then there exists a set $S:=\left\{x_{i} \in A_{i}: i \in \bar{p}\right\}$ of card $S=p$ of best proximity points of the self-map $F$ of $X$ such that $x_{i} \in \operatorname{Fix}\left(F^{p} \mid A_{i}\right)$; for all $i \in \bar{p}$, and there are Cauchy sequences $\left\{x_{i}(n)\right\}_{n=1}^{\infty}$; for all $i \in \bar{p}$ which satisfy $x_{i}(n+1)=F^{p} x_{i}(n)=$ $F x_{i-1}(n+1)$ and $x_{i}(n) \rightarrow x_{i}$ as $n \rightarrow \infty$; for all $i \in \bar{p}$. The points $x_{i}, x_{i+1}$ are $\left(A_{i} \cup\right.$ $\left.A_{i+1}\right) \times\left(A_{i} \cup A_{i+1}\right)$-close for each $i \in \bar{p}$ via some existing real constant $\delta>\bar{\alpha} g /(1-\bar{\rho})$ in Definition 2.2. Also, the pairs of Cauchy sequences $\left\{x_{i}(n)\right\}_{n=1}^{\infty},\left\{x_{i+1}(n)\right\}_{n=1}^{\infty}$ have subsequences $\left\{x_{i}(n)\right\}_{n=N}^{\infty},\left\{x_{i+1}(n)\right\}_{n=N}^{\infty}$ which are $\left(A_{i} \cup A_{i+1}\right) \times\left(A_{i} \cup A_{i+1}\right)$-close via a real constant $\delta_{0}(\varepsilon)=\varepsilon+\delta>\varepsilon+\bar{\alpha} g /(1-\bar{\rho})$ in Definition 2.2 for any given $\varepsilon>0$ and some integer $N=N(\delta, \varepsilon)$.

Proof. First note that Theorem 3.1(i)-(iii) is independent of the above modification. Note also that now $1-\alpha_{n}=\prod_{j=1}^{n}\left[1-\alpha_{j n}\right]<1$ on a subset of $\mathbf{Z}_{+}$infinite discrete measure so that (3.8)-(3.12) still hold except that $\bar{x} \in \operatorname{Fix}(F)$ is not guaranteed when $g=0$ (last part of Theorem 3.1(iv), and Corollary 3.3), since $\alpha_{j n} \equiv 1$ for $j$ belonging to some proper nonempty subset of $\bar{p}$, for all $n \in \mathbf{Z}_{+}$. It still holds that $\bar{x} \in \operatorname{Fix}\left(F^{p}\right)$. Property (i) has been proven. Now, note from Corollary 3.2 that from Theorem 3.1 there is a set $S$ of $p$ points each being a fixed point of the restricted self-map $F^{p} \mid A_{i}$, for all $i \in \bar{p}$ under the pairwise constraints

$$
d\left(x_{i}, x_{i+1}\right)=\lim _{n \rightarrow \infty} d\left(F^{n p} x, F^{n p} y\right)=\frac{g \bar{\alpha}}{1-\bar{\rho}} ; \quad \forall(x, y) \in\left(\bigcup_{i \in \bar{p}} A_{i}\right) \times\left(\bigcup_{i \in \bar{p}} A_{i}\right) ; \forall i \in \bar{p}
$$

which are necessarily in disjoint adjacent sets since the distances between all the sets are a constant $g>0$ and $F\left(A_{i}\right) \subseteq A_{i+1}$; for all $i \in \bar{p}$. Then the $A$-distance $d(x, y)$ of any pair $(x, y) \in$ $A_{i} \times A_{i+1}$; for all $i \in \bar{p}$ converges to a constant distance $g \bar{\alpha} /(1-\bar{\rho})$. Then, there is a convergent sequence $\left\{x_{i}(n)\right\}_{n=1}^{\infty}$ of points in $A_{i}$ verifying $x_{i}(n) \rightarrow x_{i}$ as $n \rightarrow \infty$ since $x_{i} \in \operatorname{Fix}\left(F^{p} \mid\right.$ $A_{i}$ ) for each $i \in \bar{p}$. Those sequences are Cauchy sequences since each convergent sequence in a metric space is a Cauchy sequence. Furthermore, $x_{i}(n+1)=F^{p} x_{i}(n)=F x_{i-1}(n+1)$ since $x_{i}(n) \in A_{i}$ implies that $x_{i} \in A_{i}, x_{i}(n+1)=F^{p} x_{i}(n) \in A_{i}$, and $x_{i+1}(n)=F x_{i}(n) \in$ 
$A_{i+1}$, for all $n \in \mathbf{Z}_{+}$, for all $i \in \bar{p}$. The remaining parts of Property (ii) concerning closeness according to Definition 2.2 follow the fact that the best proximity points of the self-map $F$ of $X$ are also fixed points of restricted composed maps to which Cauchy sequences of points converge and whose distance is $\bar{\alpha} g /(1-\bar{\rho})$. Property (ii) has been proven.

Since the validity of Theorem 3.1(iii) is independent of the modification of Condition 1 to the weaker one Condition 2 implying the use of the sequence $\left\{\alpha_{n}\right\}_{n=1}^{\infty}$ (see proof of Corollary 3.5), Condition 2 of Corollary 3.5 may be replaced with the following.

Condition 3. One has

$$
\int_{0}^{d\left(F^{(n+1) p} x, F^{(n+1) p} y\right)} \varphi(t) d t \leq\left(1-\alpha_{n}\right) \int_{0}^{d\left(F^{n p} x, F^{n p} y\right)} \varphi(t) d t+\alpha_{n} \min _{j \in \bar{p}} \int_{0}^{g} \varphi(t) d t .
$$

The above discussion may be discussed under any of the following replacements of Conditions 1-3.

Condition 4. One has

$$
\int_{0}^{d\left(F^{n p+j+1} x, F^{n p+j+1} y\right)} \varphi(t) d t \leq \psi\left(\int_{0}^{d\left(F^{n p+j} x, F^{n p+j} y\right)} \varphi(t) d t+\int_{0}^{g} \varphi(t) d t\right) ; \quad \forall j \in \bar{p} .
$$

Condition 5. One has

$$
\int_{0}^{d\left(F^{n p+j+1} x, F^{n+j+1} y\right)} \varphi(t) d t \leq \psi_{1}\left(\int_{0}^{d\left(F^{n p+j} x, F^{n p+j} y\right)} \varphi(t) d t\right)+\psi_{2}\left(\int_{0}^{g} \varphi(t) d t\right) ; \quad \forall j \in \bar{p}
$$

Condition 6. One has

$$
\int_{0}^{d\left(F^{(n+1) p} x, F^{(n+1) p} y\right)} \varphi(t) d t \leq \psi\left(\int_{0}^{d\left(F^{n p} x, F^{n p} y\right)} \varphi(t) d t+\int_{0}^{g} \varphi(t) d t\right) .
$$

Condition 7. One has

$$
\int_{0}^{d\left(F^{n p+j+1} x, F^{n p+j+1} y\right)} \varphi(t) d t \leq \psi_{1}\left(\int_{0}^{d\left(F^{n p+j} x, F^{n p+j} y\right)} \varphi(t) d t\right)+\psi_{2}\left(\int_{0}^{g} \varphi(t) d t\right),
$$

where $\psi, \psi_{1}, \psi_{2}: \mathbf{R}_{0+} \rightarrow \mathbf{R}_{0+}$ are comparison functions, namely, monotone increasing satisfying $\lim _{t \rightarrow \infty} \psi^{n}(t)=\psi_{1}^{n}(t)=\psi_{2}^{n}(t)=0$, for all $t \in \mathbf{R}_{0+}$.

Thus, $\psi(0)=\psi_{1}(0)=\psi_{2}(0)=0$ and $\psi(t)=\psi_{1}(t)=\psi_{2}(t)<t$; for all $t \in \mathbf{R}_{+}$as a consequence of their above properties to be comparison functions. In addition, $\psi: \mathbf{R}_{0+} \rightarrow \mathbf{R}_{0+}$ satisfies the subadditive condition $\psi\left(t_{1}+t_{2}\right) \leq \psi\left(t_{1}\right)+\psi\left(t_{2}\right)$. As a result of the above properties, note that: 
(a) Conditions 4 and 5 imply that

$$
\int_{0}^{d\left(F^{n p+j+1} x, F^{n p+j+1} y\right)} \varphi(t) d t \leq \int_{0}^{d\left(F^{n p+j} x, F^{n+j} y\right)} \varphi(t) d t+\int_{0}^{g} \varphi(t) d t,
$$

for all $x, y \in \bigcup_{i \in \bar{p}} A_{i}$, for all $j \in \bar{p}$ with the equality standing for some $j \in \bar{p}$ and some $x, y \in$ $\bigcup_{i \in \bar{p}} A_{i}$ if and only if $g=d\left(F^{n p+j} x, F^{n p+j} y\right)=0$, that is, the distance between relevant points in the upper-limits of the integral and between all the adjacent sets are zero.

(b) Conditions 6 and 7 imply that

$$
\int_{0}^{d\left(F^{(n+1) p} x, F^{(n+1) p} y\right)} \varphi(t) d t \leq \int_{0}^{d\left(F^{n p} x, F^{n p} y\right)} \varphi(t) d t+\int_{0}^{g} \varphi(t) d t
$$

for all $x, y \in \bigcup_{i \in \bar{p}} A_{i}$ with the equality standing for some $x, y \in \bigcup_{i \in \bar{p}} A_{i}$ if and only if $g=$ $d\left(F^{n p} x, F^{n p} y\right)=0$.

The following results follow.

Corollary 3.6. Theorem 3.1and Corollaries 3.2-3.4 hold "mutatis-mutandis" under any of the pcyclic contractive Conditions 6 and 7.

Corollary 3.7. Theorem 3.1and Corollaries 3.2-3.4 hold "mutatis-mutandis" under any of the $p$ cyclic contractive Conditions 4 and 5 except that $\bar{x} \in \operatorname{Fix}(F)$ if the distance between adjacent sets $g$ is zero (i.e., all sets $A_{i}, i \in \bar{p}$ have a nonempty intersection).

The proofs are direct as that of Theorem 3.1 (see also that of Corollary 3.5) by using the properties (3.24) for that of Corollary 3.6 and (3.23) for that of Corollary 3.7.

\section{Acknowledgments}

The author is grateful to the Spanish Ministry of Education by its partial support of this work through Grant DPI2009-07197. He is also grateful to the Basque Government by its support through Grants IT378-10, SAIOTEK S-PE08UN15, and SAIOTEK S-PE09UN12. He thanks the anonymous reviewers by their suggestions to improve the paper.

\section{References}

[1] M. Edelstein, "On fixed and periodic points under contractive mappings," Journal of the London Mathematical Society, vol. 37, pp. 74-79, 1962.

[2] A. Meir and E. Keeler, "A theorem on contraction mappings," Journal of Mathematical Analysis and Applications, vol. 28, pp. 326-329, 1969.

[3] T. Suzuki, "A new type of fixed point theorem in metric spaces," Nonlinear Analysis: Theory, Methods $\mathcal{E}$ Applications, vol. 71, no. 11, pp. 5313-5317, 2009.

[4] K. Aoyama, Y. Kimura, W. Takahashi, and M. Toyoda, "Approximation of common fixed points of a countable family of nonexpansive mappings in a Banach space," Nonlinear Analysis: Theory, Methods E Applications, vol. 67, no. 8, pp. 2350-2360, 2007.

[5] Y. Hao, "Convergence theorems of common fixed points for pseudocontractive mappings," Fixed Point Theory and Applications, vol. 2008, Article ID 902985, 9 pages, 2008.

[6] R. P. Agarwal and D. O'Regan, "Fixed point theory for admissible type maps with applications," Fixed Point Theory and Applications, vol. 2009, Article ID 439176, 22 pages, 2009. 
[7] M. O. Olatinwo, "Some common fixed point theorems for selfmappings satisfying two contractive conditions of integral type in a uniform space," Central European Journal of Mathematics, vol. 6, no. 2, pp. 335-341, 2008.

[8] S. Karpagam and S. Agrawal, "Best proximity point theorems for p-cyclic Meir-Keeler contractions," Fixed Point Theory and Applications, vol. 2009, Article ID 197308, 9 pages, 2009.

[9] M. Aamri and D. El Moutawakil, "Common fixed point theorems for E-contractive or E-expansive maps in uniform spaces," Acta Mathematica, vol. 20, no. 1, pp. 83-91, 2004.

[10] C. Chen and C. Zhu, "Fixed point theorems for $\mathrm{n}$ times reasonable expansive mapping," Fixed Point Theory and Applications, vol. 2008, Article ID 302617, 6 pages, 2008.

[11] M. De la Sen, "About robust stability of dynamic systems with time delays through fixed point theory," Fixed Point Theory and Applications, vol. 2008, Article ID 480187, 20 pages, 2008.

[12] T. A. Burton, Stability by Fixed Point Theory for Functional Differential Equations, Dover, Mineola, NY, USA, 2006.

[13] M. De la Sen and A. Ibeas, "Stability results for switched linear systems with constant discrete delays," Mathematical Problems in Engineering, vol. 2008, Article ID 543145, 28 pages, 2008.

[14] M. De la Sen and A. Ibeas, "Stability results of a class of hybrid systems under switched continuoustime and discrete-time control," Discrete Dynamics in Nature and Society, vol. 2009, Article ID 315713, 28 pages, 2009.

[15] M. De la Sen and A. Ibeas, "On the global asymptotic stability of switched linear time-varying systems with constant point delays," Discrete Dynamics in Nature and Society, vol. 2008, Article ID 231710, 31 pages, 2008.

[16] M. De la Sen, "Total stability properties based on fixed point theory for a class of hybrid dynamic systems," Fixed Point Theory and Applications, vol. 2009, Article ID 826438, 19 pages, 2009.

[17] A. Granas and J. Dugundji, Fixed Point Theory, Springer Monographs in Mathematics, Springer, New York, NY, USA, 2003. 